Supporting information for the paper

\title{
Clustering of Major Histocompatibility Complex-Class I Molecules in Healthy and Cancer Colon Cells Revealed from Their Nano-Mechanical Properties
}

Manola Moretti*†, Rosanna La Rocca $\ddagger$, Michela Perrone Donnorso $\downarrow$, Bruno Torre*†, Claudio Canale•, Mario Malerba $\ddagger$, Gobind Dasz, Rosa Sottile $\square$, Cinzia Garofalo $\square$, Adnane Achour $\square$, Klas Kärre呟, Ennio Carbone $\diamond$ ^, Enzo Di Fabrizio*§.

† Single Molecule Imaging by Light Enhanced spectroscopies Lab, King Abdullah University of Science and Technology, 23955-6900, Thuwal, Jeddah, Kingdom of Saudi Arabia

\$ Istituto Italiano di Tecnologia, Via Morego 30, 16163 Genova, Italy

- Department of Physics, University of Genova, Via Dodecaneso 33, 16146, Genova, Italy

そ Department of Physics, Khalifa University, P.O. Box: 127788 Abu Dhabi, United Arab Emirates $\square$ Katharine Hsu Lab, Memorial Sloan Kettering Cancer Center, 1275 York Avenue, New York, USA

Departement for Experimental and Clinical Medicine, University of Catanzaro, Viale Europa 88100, Catanzaro, Italy

Science for Life Laboratory, Department of Medicine, Solna, Karolinska Institute, and Division of Infectious Diseases, Karolinska University Hospital, Solna, 17176, Stockholm, Sweden.

Department of Microbiology, Tumor and Cell Biology, Karolinska Institute, Biomedicum Solnavägen 9, 17165 Solna, Stockholm, Sweden.

$\S$ Department of Applied Physics, Polytechnic University of Turin, Corso Duca degli Abruzzi, 24, 10129 Torino, ITALY 
$\diamond$ Dipartimento Medicina di Precisione, Università della Campania, via L. De Crecchio, 7, 80138 Naples, Italy

*corresponding authors: manola.moretti@kaust.edu.sa; brunotorre@gmail.com; enzo.difabrizio@polito.it

\section{Control of Antibody attachment to the AFM probe}

In order to verify the success of the functionalization process the treated AFM probes were observed with a confocal fluorescence microscope (Confocal Microscopy Upright Leica TCS SP5). Obtained images show the presence of fluorescence signal both on the apex of tip (Fig SI1a) and on the cantilever (Fig. SI1b). The typical spectrum of Alexa 488 was found (Fig. SI1) indicating the presence of the purified SAT(PEG) $)_{4}$ modified Alexa Fluor 488 goat anti-mouse antibody.

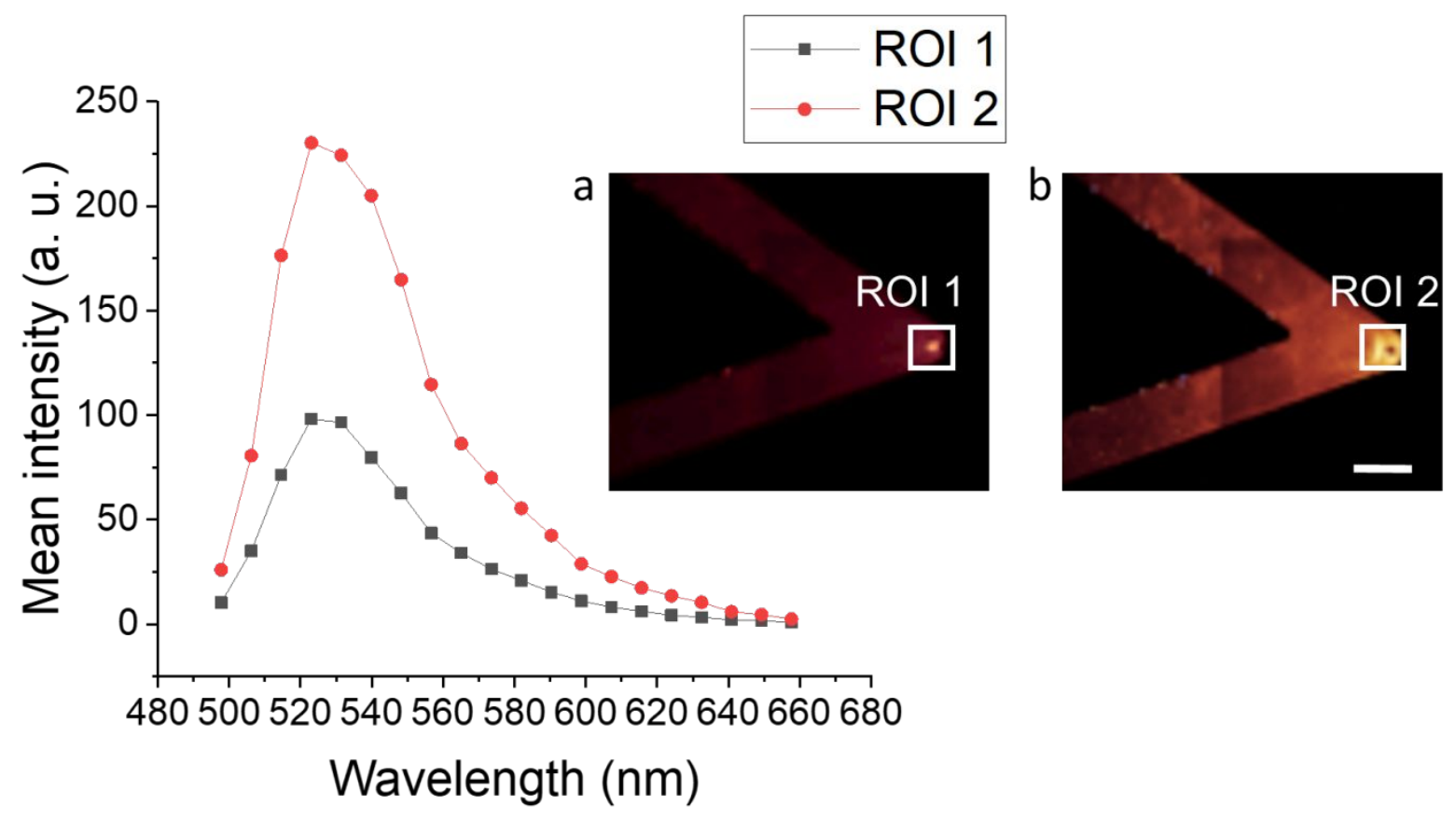


Figure SI1: Confocal microscopy measurements of AFM probe after the functionalization process. Fluorescence signal from the tip apex (a) and the cantilever (b)); the plot shows the intensity results related to the spectrum of the Alexa 488 fluorophore.

\section{Controls for the effectiveness of the experimental procedure}

Fluorescence and optical image

The presence of active MHC-I sites was verified after AFM force spectroscopy mapping by applying a secondary antibody with fluorescent tag in the petri dish containing the cells. Images were acquired directly in the JPK AFM microscope in standard optical imaging configuration, equipped with a Photofluor II lamp (200W, self-aligning metal-halide lamp, Chroma, 89 North Inc.). The fluorescent images overlaid to the bright field image of the cells show that the MHC-I molecules are still active for the interaction with the secondary antibody, thus available for further analysis (Fig. SI2).
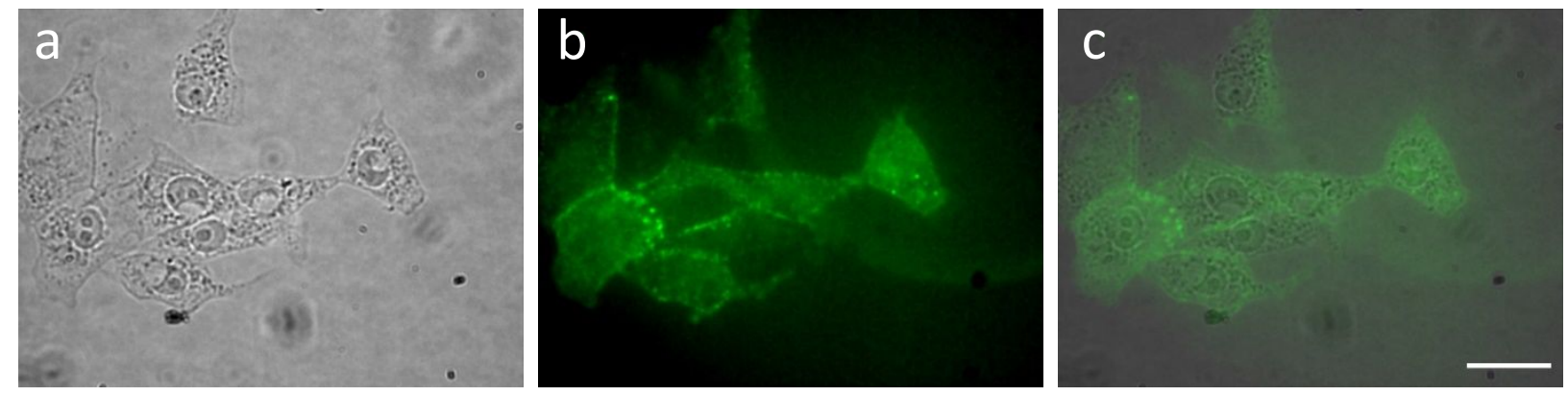

Figure SI2: Secondary fluorescent antibody tagging of the MHC-I binding sites after AFM force spectroscopy. Optical (a), fluorescence (FITC) (b) and overlapped images (c) of the HCT cells after treatment with fluorescence antibody (W6-32) tagging MHC-I molecules. Scalebar $20 \mu \mathrm{m}$. 


\section{Controls for the effectiveness of the procedure with AFM}

To reassure that the pulling was specific of the molecule of interest, we verified that the number of pulling events went down to almost zero after addition of the blocking antibody in the buffer. The experiment consist of performing the force spectroscopy measurements on 3 cells for each sample, blocking the membrane surface by introducing a suitable amount of secondary antibody and acquiring force maps with the same used tip and with a new functionalized one.

The saturation of the binding sites of the primary antibody attached to the MHC-I protein, is effectively limiting the pulling events from occurring, both with the used tip and the freshly functionalized tip. In Fig. SI3 the comparison between average area values (expressed as percentage on absolute value) underneath the curves of about 100 pulling events obtained from experiment series are shown. The area value of MHC-I pulling from HCT cancer cells is significantly higher (93\%) than area values of the negative controls (less than 3\%).

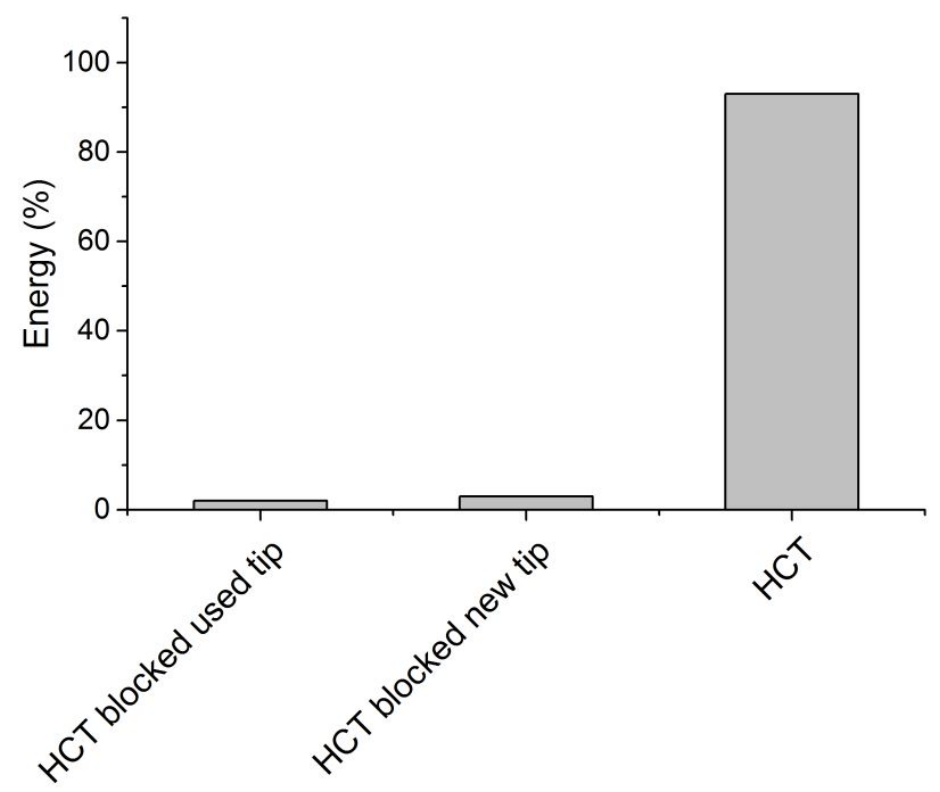


Figure SI3: Effect of introduction of blocking antibody on the force-distance AFM curves on HCT cells. Comparison of average Energy of adhesion values (expressed in percentage) underneath the retract curve of 100 representative force spectroscopy curves for: pulling of MHC-I pulling after blocking with secondary antibody, pulling after blocking with secondary antibody on a fresh functionalized tip, and pulling from HCT cell membrane before blocking.

Control that MHC-I is not stripped from the cell membrane and of the reproducibility of Energy of adhesion values

See description in the main text

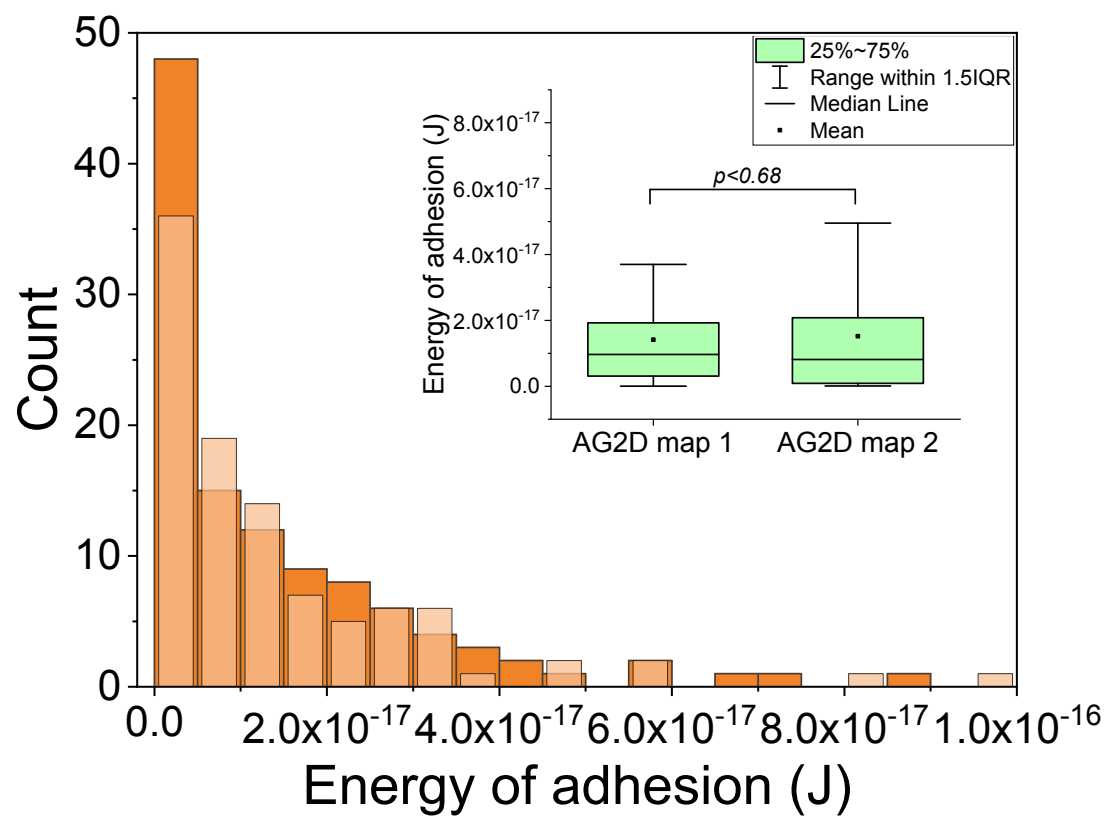

Figure SI4. Evaluation of reproducibility of Energy of adhesion values per pixel by overlapping of map measurements performed on the same cell, with the same AFM probe. The two population are significantly different only at $p<0.68$. 


\section{Bottom effect on calculation of Young's modulus}

See main text and Materials and Methods for detailed explanation

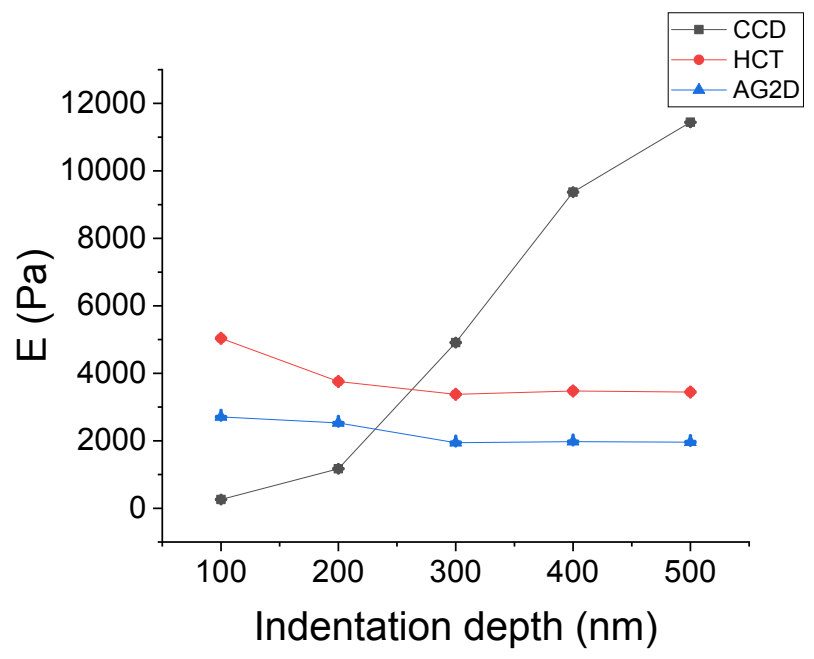

Figure SI5: Evaluation of the bottom effect on the Young's modulus at different indentation depths for the three cell types.

\section{AFM tip modeling for specific and unspecific interaction calculations}

In the reported AFM Force-Distance (FD) experiments, the average number of binding events $<n>$ per unit area is calculated for each loading and unloading from the measurement of the interaction energy, the separate measurement of the unspecific (e.g. van der Waals, electrostatic interactions) surface energy density and the evaluation of the contact area at the maximum load of each loading curve. This last is evaluated form the fit of the loading part of the curve, using a simplified geometrical mode of the tip, usually depicted as a paraboloid or a sphere in the very apical (mostly used in low-loads regime) or sharper geometries such a pyramid or cones in case of very sharp tips or higher indentations. Here, we refine the Hertz-Sneddon model to account for a spherical apical tip of arbitrary radius $R$ on cones/pyramids of semi-aperture $\alpha$, to be used in the general case. We 
analytically compute and display the correction factor (multiplicative) applied with respect to the ideally sharp case for our tip parameters, to visualize the entity of the correction at different indentation depth $h$. The advantage of displaying the adjustment as a pre-factor is that all the relevant parameters calculated from the Hertz-Sneddon model can be corrected afterwards in a trivial way. As expected, the factor tends to 1 in the limit of $R \rightarrow 0$ and/or $h \rightarrow \square$. Finally, we use this correction to give a corrected expression of $<\mathrm{n}>$.

\section{Hertz-Sneddon for ideally sharp tips}

The contact area $A$, in the case of square base pyramid and cone, is written as:

$$
\begin{gathered}
A_{\text {pyr }}=\frac{P_{\text {Max }} \pi \sqrt{\pi}}{E^{*} \cos \alpha} \\
A_{\text {cone }}=\frac{P_{\text {Max }} \pi^{2} \sqrt{\pi}}{4 E^{*} \cos \alpha}
\end{gathered}
$$

Where $P_{M a x}$ is the maximum load applied at the end of the approach curve, $E^{*}=E /\left(1-v^{2}\right)$ is the simplified reduced Young's modulus, with $E$ the Young's modulus for sample elasticity and $v$ the Poisson ratio of the sample ( 0.5 was used in this case) and $\alpha$ is the pyramid or cone half angle.

\section{Hertz-Sneddon for rounded tips.}

The model takes into account the case of a rounded tip, modeled as a sphere cap of radius $R$, under the geometrical constrain that the tangent to the sphere in the connection point has the same tilt as $\alpha$, i.e. lays on the side of the cone or pyramid, as depicted in fig SI6a. 

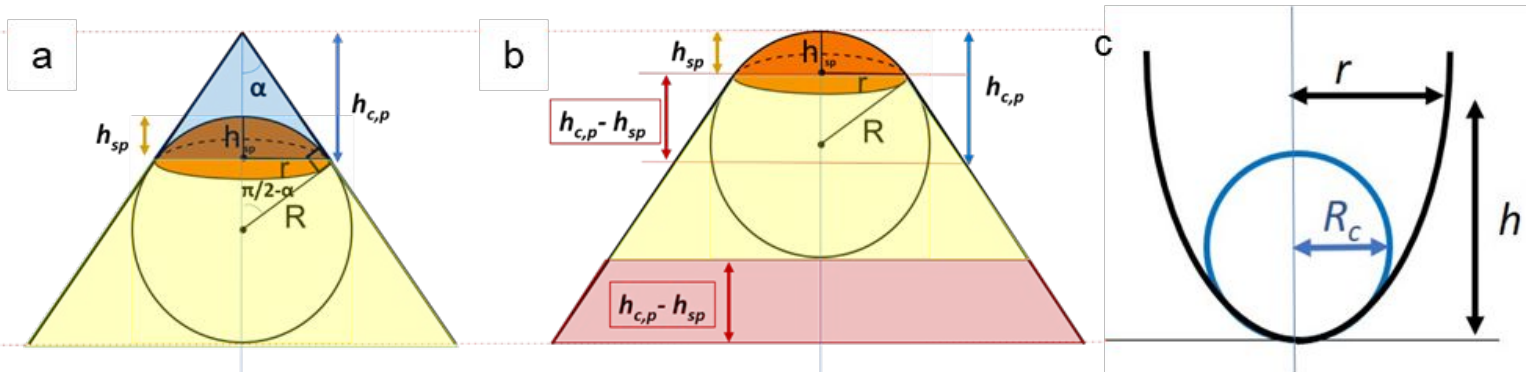

Figure SI6. a) Geometrical model of the spherical cap (orange) replacing the ideally sharp conical or pyramidal apex of the tip. Continuity condition is established setting the spherical cap tangent to the cone or to the center of the pyramid faces in the connection point. b) Geometrical configuration of the rounded tip at the same contact point (dotted red line). For the same indentation, an additional portion of the truncated cone/pyramid (red) enters in contact with the sample.

The introduction of a geometrical rounded tip has 2 main effects: 1) the area of the ideally sharp apex (blue) is replaced by the area of the spherical cap (orange) (fig S1.a) and, 2) at the contact point the truncated cone/pyramid part moves forward by the height difference between the sharp and the rounded apex, as

$$
\Delta h=h_{c, p}-h_{s p}=R\left(\frac{1}{\sin \alpha}-1\right) ; \quad \text { with } \quad h_{s p}=R(1+\sin \alpha)
$$

For indentations $h<h_{s p}$ the indentation area is the spherical cap one $A_{s p, c a l}=2 \pi R h$, while for $h>h_{s p}$ the contact area of equations S1.a and S1.b is rewritten as

$$
\begin{aligned}
& A_{\text {cone }, \text { cap }}=\pi(h+\Delta h)^{2} \frac{\tan \alpha}{\cos \alpha}-\pi h_{c}^{2} \frac{\tan \alpha}{\cos \alpha}+2 \pi R h_{s p} \\
& A_{\text {pyr,cap }}=4(h+\Delta h)^{2} \frac{\tan \alpha}{\cos \alpha}-4 h_{p^{2}}^{2} \frac{\tan \alpha}{\cos \alpha}+2 \pi R h_{s p}
\end{aligned}
$$

Where $h_{c, p}$ is the height of the cone or pyramid respectively.

If we define 


$$
\Delta_{c}=\frac{A_{\text {cone, cap }}}{A_{\text {cone }}}
$$

and

$$
\Delta_{p}=\frac{A_{\text {pyr }, \text { ap }}}{A_{\text {pyr }}}
$$

The area in eq S1.a,b can be rewritten as

$$
\begin{gathered}
A_{\text {pyr,cap }}=\frac{P_{\text {Max }} \pi \sqrt{\pi}}{E^{*} \cos \alpha} \Delta_{p} \\
A_{\text {cone }, \text { cap }}=\frac{P_{\text {Max }} \pi^{2} \sqrt{\pi}}{4 E^{*} \cos \alpha} \Delta_{C}
\end{gathered}
$$

Figure SI7 sums up the results

(a)

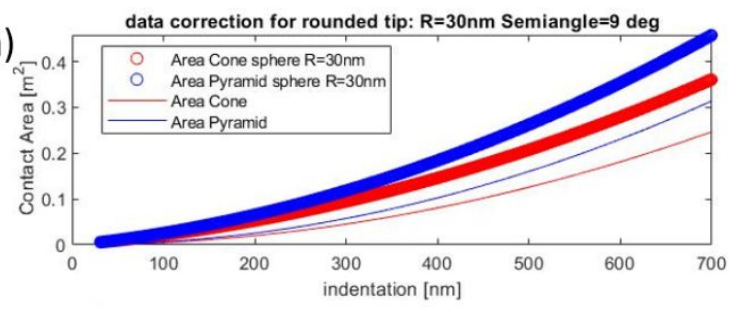

(c)

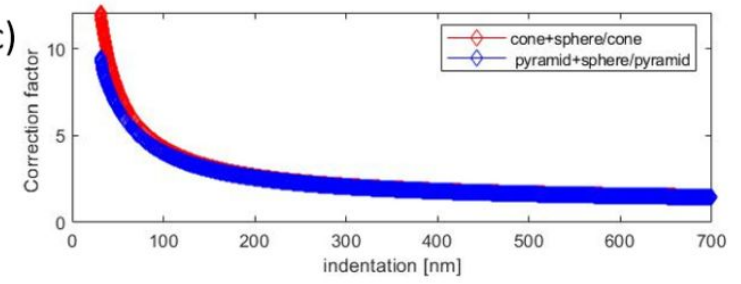

(e)

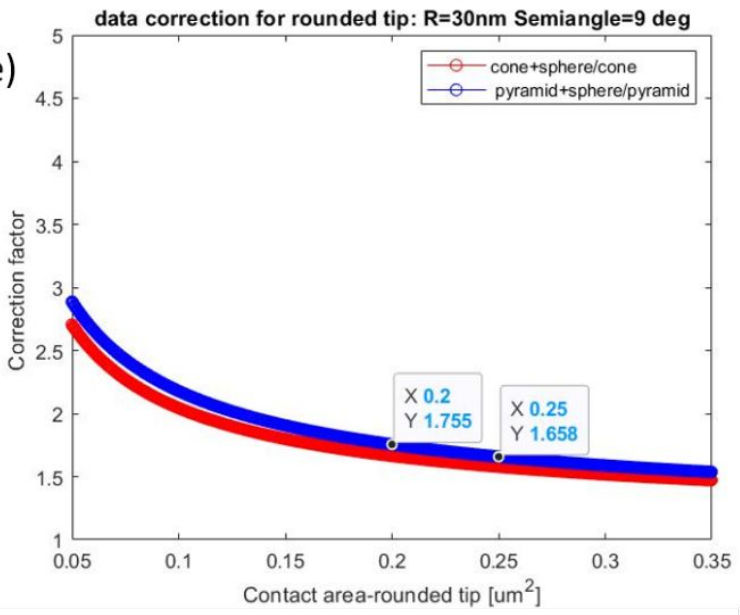

(b)

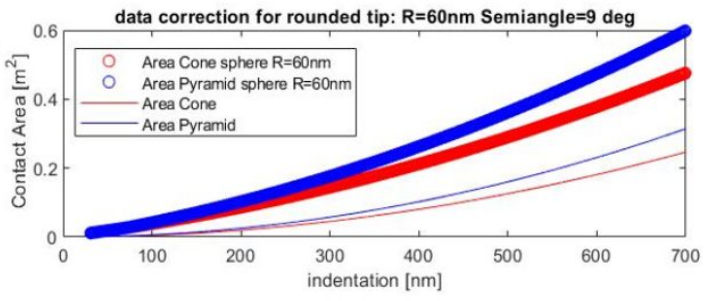

(d)
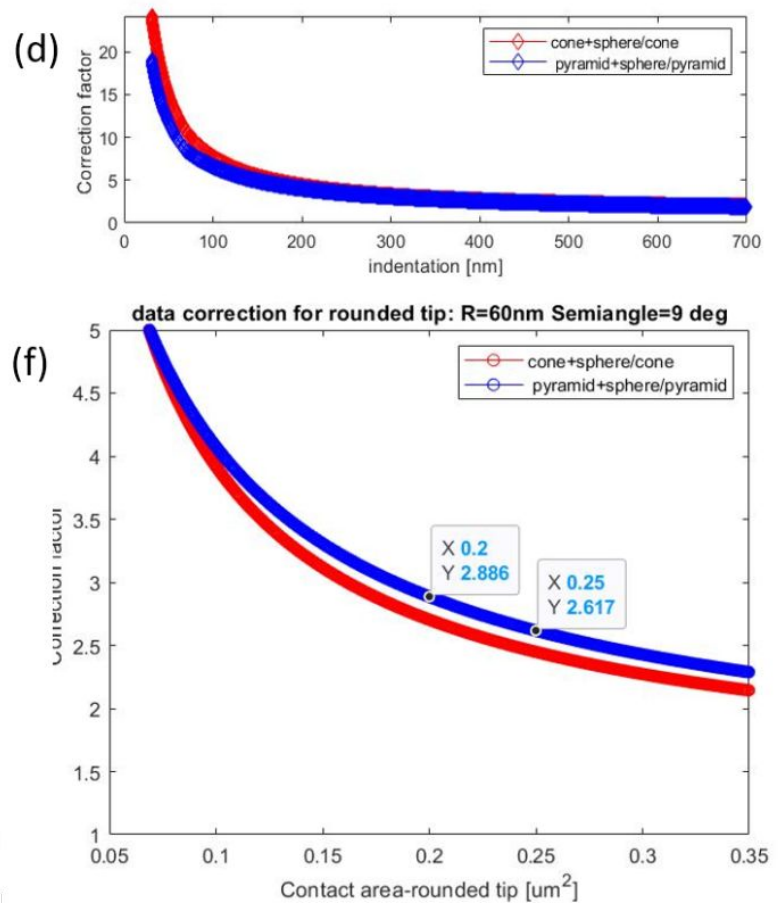

Figure SI7. The figure displays the results of the variation in contact area, and delta parameters from equations S3.a,b and S4.a,b as function of indentation depth and contact area 
calculated for $\alpha=9^{\circ}$ and $R=30 \mathrm{~nm}$ and $60 \mathrm{~nm}$. More specifically: a) Contact area vs indentation depth for $R=30 \mathrm{~nm}$ and (b) $R=60$, sharp pyramid (blue line), sharp cone (red line), rounded tip pyramid (blue circles) rounded tip cone(red circle); c) Correction factor vs indentation depth for $R=30 \mathrm{~nm}$ and (d) $R=60$, sharp pyramid (blue line), sharp cone (red line), rounded tip pyramid (blue circles) rounded tip cone(red circle); e) Correction factor vs contact area (rounded tip model) for $R=30 \mathrm{~nm}$ and (f) $R=60$, sharp pyramid (blue line), sharp cone (red line), rounded tip pyramid (blue circles) rounded tip cone(red circle).

In the same way, the number of bindings per unit area $\langle n>$, calculated from the expression of the adhesion energy

$E_{\text {adhesion }}=\gamma_{A} A_{c}+\langle n\rangle E_{b} A_{c}$

Is modified from the ideal cone case, as obtained from equations S1.a, S1b and S6,

$\langle n\rangle=\frac{1}{E_{b}}\left(\frac{E_{\text {adhesion }}}{P_{\max }} * \frac{E^{*} \cos \alpha}{\pi \sqrt{\pi}}-\gamma_{A}\right)$

for the pyramidal probe and,

$\langle n\rangle=\frac{1}{E_{b}}\left(\frac{E_{\text {adhesion }}}{P_{\text {Max }}} * \frac{4 E^{*} \cos \alpha}{\pi^{2} \sqrt{\pi}}-\gamma_{A}\right)$

(S7.b), for the

conical probe,

to the form

$\left\langle n_{p}\right\rangle=\frac{1}{E_{b} \Delta_{p}}\left(\frac{E_{\text {adhesion }}}{P_{\text {Max }}} * \frac{E^{*} \cos \alpha}{\pi \sqrt{\pi}}-\gamma_{A}\right)$

And

$\left\langle n_{c}\right\rangle=\frac{1}{E_{b} \Delta_{c}}\left(\frac{E_{\text {adhesion }}}{P_{\text {Max }}} * \frac{4 E^{*} \cos \alpha}{\pi^{2} \sqrt{\pi}}-\gamma_{A}\right)$

Respectively, which contains the correction parameter. 
Again, in the limit $R \rightarrow 0$, the correction parameters tend to 1 in both cases and equations S8 and $\mathrm{S} 7$ coincide.

\section{Paraboloid tip}

In case the tip can be described by a paraboloid shape (Fig. SI6c), the contact area can be analytically described in terms of the disk radius $r$ at the indentation distance $h$ from the tip, as

$A_{\text {par }}=\frac{\pi r}{6 h^{2}}\left[\left(r^{2}+4 h^{2}\right)^{\frac{3}{2}}-r^{3}\right]$

If $R_{c}$ is the curvature radius of the tip, then $r=\left(2 R_{c} h\right)^{\frac{1}{2}}$. It is therefore possible to express the contact area as function of the indentation depth and geometrical parameters only, as

$A_{\text {par }}=\frac{2 \pi R_{c}^{2}}{3}\left[\left(1+\frac{2 h}{R_{c}}\right)^{\frac{3}{2}}-1\right]$

In the Hertz-Sneddon model the indentation depth $h$, reduced Young modulus $E^{*}=E /\left(1-v^{2}\right)$ and the maximum load $P_{\operatorname{Max}}$ are related as

$P_{\text {Max }}=\frac{4}{3} \sqrt{R_{c}} E^{*} h^{\frac{3}{2}}$

By combining s10 and s11, we finally get

$A_{\text {par }}=\frac{2}{3} \pi R_{c}^{2}\left\{\left[1+\left(\frac{9 P_{\operatorname{Max}}^{2}}{2 E^{* 2} R_{c}^{4}}\right)^{\frac{1}{3}}\right]^{\frac{3}{2}}-1\right\}$

Combining S6 and S12 we finally get

$$
\left\langle n_{\text {par }}\right\rangle=\frac{1}{E_{b}}\left(\frac{3 E_{\text {adhesion }}}{\left.2 \pi R_{c}^{2}\left[1+\left(\frac{g^{2}}{2 E^{*} R_{R_{c}^{4}}^{4}}\right)^{\frac{1}{3}}\right]^{\frac{3}{2}}-1\right)}-\gamma_{A}\right)
$$




\section{Fabrication of the nanocone probe and experimental part to verify equation (3.c)}

Devices were fabricated on commercial AFM cantilevers (NPG-10, Bruker AFM Probes). AFM cones were grown using a FEI Helios Nanolab 600, by electron beam induced deposition from a platinum gas precursor. A reproducible tapered shape was achieved by finely tuning beam parameters and pattern geometries. The apex diameter of $30 \mathrm{~nm}$ was then coated with metal by sputtering a uniform gold layer of $30 \mathrm{~nm}$, thus increasing the apex to a diameter of $60 \mathrm{~nm}$. To avoid any carbonaceous contamination, imaging was not performed on specimen prior to gold functionalization. Fig. SI8a shows an example of cone. We performed the same measurement of force spectroscopy with nanocone probe functionalized with the secondary antibody, solely on HCT cells. The measurements were performed both with the nanocone with a ROC of $30 \mathrm{~nm}(9$ cells investigated) and $60 \mathrm{~nm}$ ( 4 cells investigated). Afterwards we calculated the number of MHCI molecules per $\mu \mathrm{m}^{2}$ (Fig. SI8b) for each type of probe that display very similar values, not statistically different. Assuming that the population of HCT is self-similar, with this test we showed that the equation (3.b) can be applied to different types of probe geometry with meaningful results. 

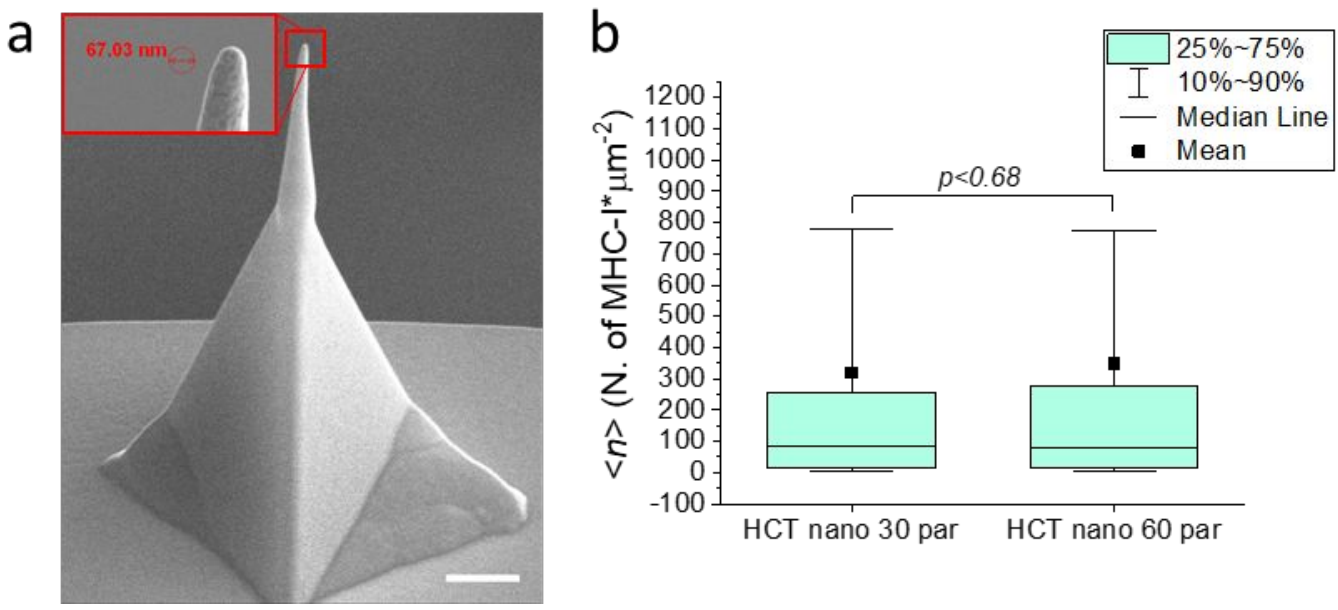

Figure SI8: Overview of nanocone by SEM imaging. a, SEM micrograph of the nanocone fabricated on commercial silicon nitride pyramid probe, coated with gold, with a radius of curvature (ROC) of about $30 \mathrm{~nm}$ (inset in a); scalebar $1 \mu \mathrm{m} ; \mathrm{b}$, comparison of number of recognition events $N$. MHC/ $\mathrm{m}^{2}$ on HCT cells, calculated with equation 3.b applied to the two different geometries of the nanocone probe (ROC $30 \mathrm{~nm}$ and ROC $60 \mathrm{~nm}$ ). Measurements performed on HCT cells with the nanocone probe and the Young's modulus is calculated by applying the Hertz model for a paraboloid probe.

\section{Pearson's correlation coefficient}

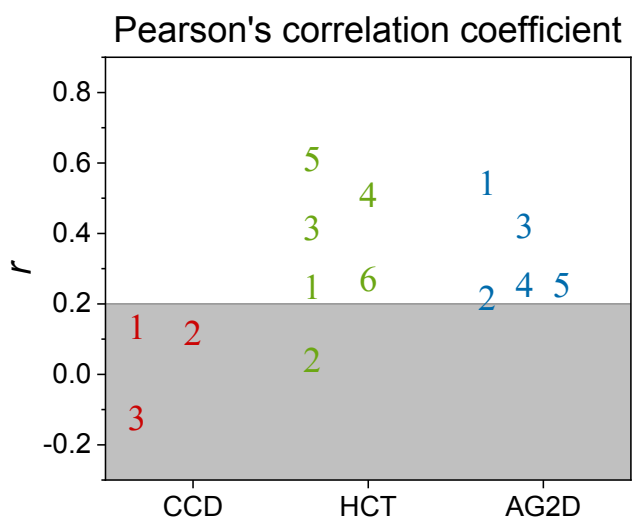

Figure SI9: Pearson's correlation coefficient (r) indicating linear correlation between the Young's modulus and the N. MHC-I molecules, for each cell per each cell type (single cells are indicated by a number). 


\section{Morphological comparison of the cells at the micro scale by AFM topography}

Morphological differences between CCD cells and HCT cells can be visualized in the following representative AFM images. Unfortunately we were not able to perform topography measurement on the AG2D cell lines because they were very loosely attached to the Petri dish, and therefore the drag force of the probe was enough to displace them. In Fig. SI6a, c 2D and 3d images of a single CCD cell grown in adhesion on a petri dish is shown. The body of the cell appears well adherent on the substrate being well spread on it. Many extensions of the cell depart from its margins, both for anchoring to the surface and for colonizing new areas. Fine structures corresponding to the cytoskeleton in the inner layer are evident. The cytoskeleton gives the cell its spread shape and in the meantime provides support for the fine structures to reach out for new territories. In Fig. SI6b, d $2 \mathrm{D}$ and $3 \mathrm{~d}$ images of two cells of the HCT population are plot and show a quite different phenotype compare to CCD. The cells appear not spread on the substrate, bumpy, and with very few propagule. The underneath cytoskeleton is not evident were instead several microvilli appear from the surface, which is quite wavy itself.
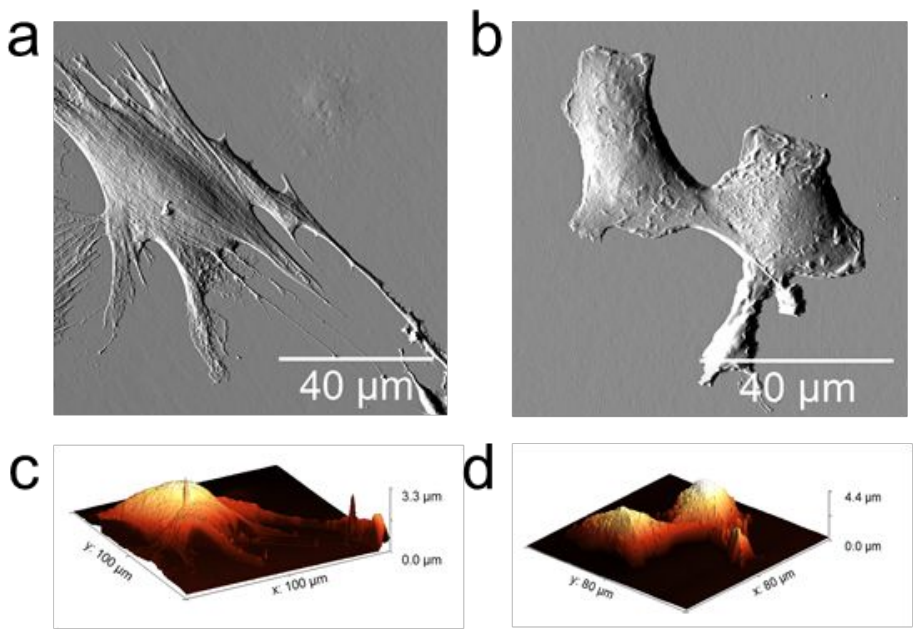

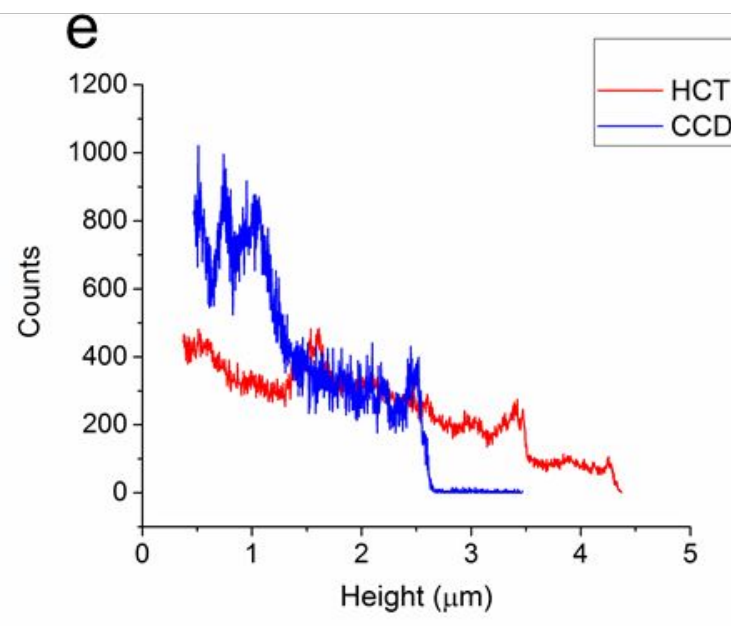


Figure SI10: Topography images and plot of height distribution of CCD and HCT cells. AFM amplitude signal of CCD (a) and HCT cells (b). $3 D$ view of the AFM topography of CCD (c) and $H C T(d)$. Height distribution retrieved from AFM topography image of a single CCD and HCT cell.

Some micro-, nano-scale properties were retrieved by performing the following analysis. The histogram of the height distribution for the couple $\mathrm{CCD} / \mathrm{HCT}$, is presented in Fig. SI6e. We can observe that the healthy epithelial colon cells present a height distribution with 3 peaks: one at around $700 \mathrm{~nm}$, one at around $1 \mu \mathrm{m}$ and one at around $2.5 \mu \mathrm{m}$. We can attribute the larger height value to the nucleus presence, the medium height to the body of the cell and the smallest height to the flat protrusions of the cell spreading on the surface. The cancer cell HCT has a similar pattern but it is slightly shifted to larger height values such that the maximum peak is around 3.0 to 3.5 $\mu \mathrm{m}$, the medium one at $1.0-1.5 \mu \mathrm{m}$ and the smallest one at $0.5 \mu \mathrm{m}$. This confirms that there are very few protrusions of the HCT cells on the substrate and that these cells are quite "bumpy" and may have not adhered firmly to the substrate. This is a typical characteristic of these cells that was also observed under optical microscope and supports the observation made by looking at the AFM topography images.

In Table SI1, statistical quantities related to the cell surface microscale properties are reported. The first observation that can be made is that the height quantities (average, maximum and median height values) of the wild type cell line $\mathrm{CCD}$, for healthy colon cell, is significantly less than the one of the corresponding cancer type cell HCT. Then, by comparing the surface area with the projected area, we observe that the projected area for the cancer type cells (HCT) is significantly lower than the healthy type cells (CCD). This is an indication, again, that the cells do not adhere well to the culture substrate. The analysis of the roughness quantities follows. The roughness calculation depends on parameters such as scale and sampling frequency of the topography images. In these experiments, the pixel size is around $200 \mathrm{~nm}$, such that it is not possible to discriminate 
smaller features in the profile, or the feature will be affected by sampling rate ${ }^{1}$. In this case the only parameter that can be calculated here is the waviness of the surface which is computed by the roughness value upon choosing the correct cut-off and it is enough interesting because it gives information on the presence of microvilli that characterize the cell shape. Roughness values are higher for HCT as compared to its counterparts CCD. This means that microvilli structures are more represented in these cells. From comparing the average roughness ( $\mathrm{Ra})$ with the root mean square roughness (Rms) we can see that Rms values are higher, which means that there are large deviations from the mean height value. Skewness and Kurtosis values describe the distribution of the sample height data. In this case the Skew negative value indicates that there are more valleys than peaks in each sample analyzed. The low values of Kurtosis $(<3)$ testify that there are many moderate height features. The fact that the wild type colon cell possess a slightly higher value of Kurtosis, might indicate the sharpness of the profile interested by cytoskeletal features, which also emerges in the AFM topography.

Table S1: statistical quantities of nanoscale properties of the membrane surface emerging from topography of single HCT and CCD cell.

\begin{tabular}{|l|l|l|l|l|l|}
\hline Statistics on & CCD841 CoN & HCT116 & Statistics & CCD841 & HCT116 \\
on & CoN & \\
\hline Average value (nm) & 2434.000 & 3863.000 & Kurtosis & 1.380 & -0.043 \\
\hline Minimum (nm) & 2142.000 & 2737.000 & $\begin{array}{l}\text { Surface } \\
\text { area }\left(\mu m^{2}\right)\end{array}$ & 104.000 & 107.000 \\
\hline Maximum (nm) & 2625.000 & 4378.000 & $\begin{array}{l}\text { Projected } \\
\text { area }(\mu m 2)\end{array}$ & 103.000 & 103.000 \\
\hline Median (nm) & 2445.000 & 3886.000 & $\begin{array}{l}\text { Variation } \\
(\mu m 2)\end{array}$ & 14.000 & 27.000 \\
\hline Ra & 61.000 & 232.000 & Inclination & 1.000 & 1.600 \\
\hline
\end{tabular}




\begin{tabular}{|l|l|l|l|l|l|}
\hline & & & $\theta$ & & \\
\hline Rms & 81.000 & 288.000 & $\begin{array}{l}\text { Inclination } \\
\varphi\end{array}$ & -175.400 & -171.800 \\
\hline Skew & -1.000 & -0.555 & & & \\
\hline
\end{tabular}

\section{Local plasticity calculations and plots}

Finally, we wanted to evaluate the local Energy dissipation of the cell membrane in relation to the recognition number of MHC-I molecules (Fig. 1d). The cell is a viscoelastic body and can therefore react plastically and/or elastically to an applied pressure, even after fixation. The dissipation energy in a F-D curve is identified as the area enclosed by the approach and retract curves. We expect that the plasticity would be modified similarly to the Young's Modulus by the presence of clusters of MHC-I molecules. The Energy dissipation was evaluated by calculating the plasticity index value, as previously described ${ }^{2}$. For this analysis, only the cells with positive $r$ were chosen. We found no apparent correlation $(r)$ between the number of pulling events and the plasticity of the cells locally (results not shown). This could be an effect of the fixation of cells and of the 0.2 seconds of permanence of the probe on the cell surface. In this time lapse, the cell membrane would adapt to the force applied by the probe, and may lose some of the information required. Another reasonable explanation for this apparent discrepancy is the fact that the analysis of the plasticity takes into account the integral of the energy dissipation area, while the Young's modulus is calculated based on the derivative calculated at the onset point of the approach curve, therefore less sensitive to steep variations. Nonetheless significant differences in the plasticity among the 
cells was found, where the HCT and AG2D cells are significantly more plastic compared to CCD cells, and the CCD cells are more elastic (Fig. SI11). Moreover the peak centers of the data distributions for each cell have a striking match in their values (Fig. SI11 a, b, c), and the different area distribution of these peaks is causing the disparity in the average plasticity indexes among the 3 cells types (Fig. SI11d). These results are in good agreement with the Young's modulus average values calculated before (Fig. 2b). Index of plasticity values in the range $0.15-0.20$ are in agreement with the literature where a stiffening of the cell was observed after treatment with the fixative glutaraldehyde ${ }^{2}$.
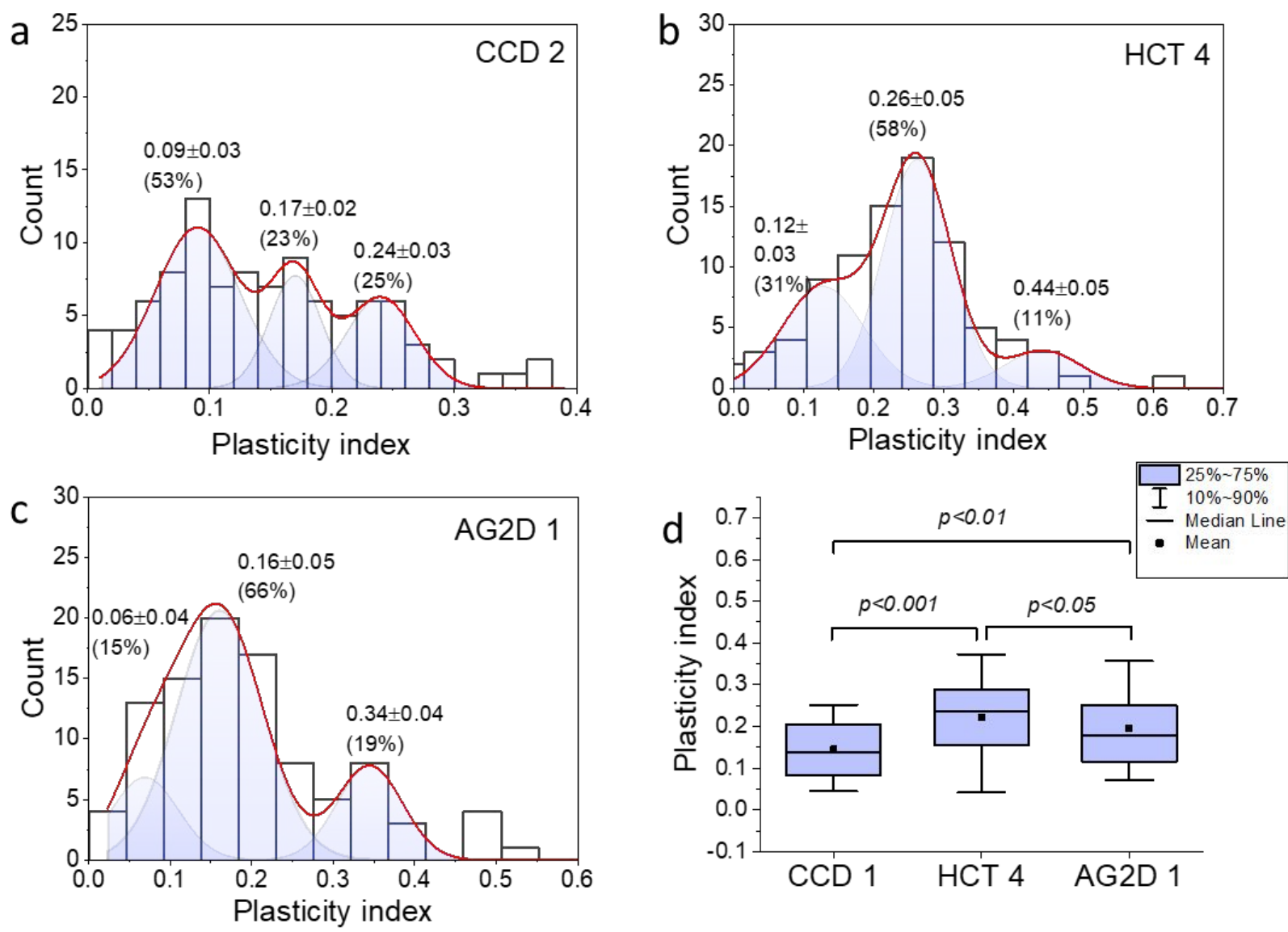

Fig. SI11: Plasticity index values for CCD, HCT and AG2D (the associated numbers refer to the cell index number) with positive Pearson correlation coefficient. a, b, c histograms of the plasticity 
index distribution with components curve fitting and related values. $d$, Box plots for population comparison. Statistical analysis as per Non-parametric Kruskal-Wallis ANOVA and post-hoc test.

\section{REFERENCES}

1. Antonio, P. D.; Lasalvia, M.; Perna, G.; Capozzi, V., Scale-Independent Roughness Value of Cell Membranes Studied by Means of AFM Technique. Biochim. Biophys. Acta 2012, 1818 (12), 3141-8.

2. Klymenko, O.; Wiltowska-Zuber, J.; Lekka, M.; Kwiatek, W., Energy Dissipation in the AFM Elasticity Measurements. Acta Physica Polonica-Series A General Physics 2009, 115 (2), 548. 